\title{
A Study on Temperature Compensation during Sleep Onset with Sleep Stage Analysis
}

\author{
Jongsoo Kum ${ }^{1}$, Donggyu $\mathrm{Kim}^{2}$, Minsoo $\mathrm{Kim}^{3}$, and Yonghyun Chung ${ }^{4}$
}

\begin{abstract}
Due to hot and humid climate and the heat island effect during summer days, tropical night phenomena frequently occur in large cities. A hot and humid environment disturbs sleep, makes it difficult for people to sleep and reduces sleep quality enough that people are rendered incapable of recovering from fatigue. If a human body is exposed to a hot and humid environment at sleep onset, slow-wave sleep (SWS) is restricted and with increased exposure time, the duration of wakefulness becomes increased, resulting in delaying the drop time of core body temperature. Therefore, to induce comfortable sleep during a tropical night, it is necessary to make sleeping easier by providing a cool environment during sleep onset. In this study, a temperature compensation condition $\left(24^{\circ} \mathrm{C} \sim 28^{\circ} \mathrm{C}\right)$, with different temperature increasing modes used to prevent hypothermia after an initial temperature $\operatorname{drop}\left(24{ }^{\circ} \mathrm{C} \sim 28{ }^{\circ} \mathrm{C}\right)$ to provide coolness during sleep onset was proposed. The sleep experiment was carried out inside an environmental chamber. The subjects were 4 males and 4 females and a quantitative assessment was performed for temperature compensation condition with classified sleep stages.
\end{abstract}

Keywords-Comfort sleep, Sleep stage, Sleep efficiency, Total sleep time

\section{INTRODUCTION}

D UE to hot and humid climate and the heat island effect during summer days, tropical night phenomena frequently occur in large cities. A hot and humid environment disturbs sleep, makes it difficult for people to sleep and reduces sleep quality enough that people are rendered incapable of recovering from fatigue.

Kazue Okamoto-Mizuno et al. reported that if a human body is exposed to a hot and humid environment during onset of sleep, SWS is restricted and with increases in the exposure time, the wakefulness stage is increased and the dropping time of the core body temperature becomes delayed, but these phenomena do not occur if a human body is exposed to a hot and humid environment during the later stages of sleep[1]. Therefore to induce comfortable sleep during tropical nights, it is necessary

Jongsoo Kum ${ }^{1}$ is with the Department of Refrigeration \& Air Conditioning Engineering, Pukyong National University, (48547) 365, Sinseon-ro, Nam-Gu, Busan, Korea

Donggyu $\mathrm{Kim}^{2}$ is with the Department of Specialized Graduate School Science \& Technology Convergence, Pukyong National University, (48547) 365, Sinseon-ro, Nam-Gu, Busan, Korea.

Minsoo $\mathrm{Kim}^{3}$ is with the Graduate School of Refrigeration \& Air Conditioning Engineering, Pukyong National University, (48547) 365, Sinseon-ro, Nam-Gu, Busan, Korea.

Yonghyun Chung ${ }^{4}$ is with the Department of Ecological Engineering, Pukyong National University, (48513) 45, Yongso-ro, Nam-Gu, Busan, Korea. to provide a cool environment during the onset of sleep to induce sleep easily. In preceding research, an optimum room temperature of $24{ }^{\circ} \mathrm{C}$ during sleep onset was proposed, but considering mean skin temperature, survey result etc., the necessity of temperature increase during sleep and its upper limit were suggested[2,3,4,5,6]. A low air temperature means cold stress to human, which has more negative effects on the sleep quality than heat stress[7]. Temperature compensation condition was proposed in this study in order to figure out the effect of room temperature change during sleep onset and it also attempted to provide basic data for air conditioning processes used to induce comfortable sleep by carrying out a qualitative assessment of temperature compensation conditions based on sleep stages.

\section{EXPERIMENT METHOD}

\section{A. Experiment Condition}

After ventilating the sleeping room sufficiently and maintaining the room temperature at $28^{\circ} \mathrm{C}$, the relative humidity was raised to $50 \%$ by using a thermo-hygrostat by running it for 2 hours prior to the experiment, in order make room's conditions the same as those of a tropical night. According to related research, if a low temperature of $24^{\circ} \mathrm{C}$ is maintained during sleep onset, mean skin temperature is also maintained at a low level which is outside of the mean comfortable mean skin temperature range[3, 6]. In this study, in order to figure out the effects of two sets of temperature increase conditions on sleep i.e., Case A $\left[4{ }^{\circ} \mathrm{C} / 0.5 \mathrm{~h}\right]$ and Case B $\left[4{ }^{\circ} \mathrm{C} / \mathrm{h}\right]$ during the temperature compensation period, followed by a temperature drop $\left(28^{\circ} \mathrm{C} \rightarrow 24^{\circ} \mathrm{C}\right)$ after sleep onset were compared. Total course of this experiment was 8 hours, with a sleep onset time of 120 minutes being set as analysis range in this study. Fig. 1 shows room temperature changes during the 120 minutes of sleep onset, after temperature the compensation process in Case A and Case B during which a thermo-hygrostat was used to regulate temperature. Case $\mathrm{A}$ is the condition in which increasing the temperature to $28^{\circ} \mathrm{C}$ in 30 minute's time was carried out during the temperature compensation section (60min) and maintained at this temperature. While Case B was the condition in which the temperature was increased gradually to $28{ }^{\circ} \mathrm{C}$ through the 60 minute period of the temperature compensation section after sleep onset. 


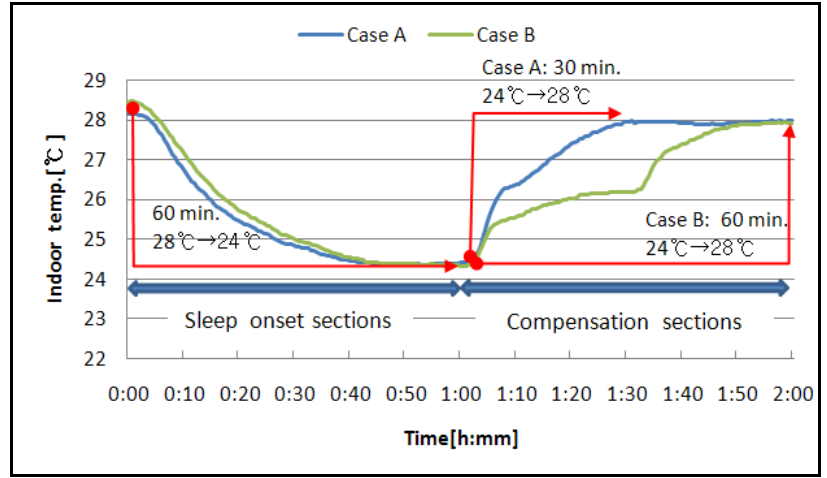

Fig. 1 Temperature compensation control process

TABLE I

ANTHROPOMETRIC DATA OF THE SUBJECTS

\begin{tabular}{ccc}
\hline \hline Sex & Male & Female \\
\hline Number of subjects & 4 & 4 \\
Age[year] & $25.0 \pm 1.4$ & $22.0 \pm 0.8$ \\
Height[cm] & $172.3 \pm 5.6$ & $162.6 \pm 2.5$ \\
Weight[kg] & $66.0 \pm 6.97$ & $47.8 \pm 3.77$ \\
Pulse & $74.8 \pm 11.1$ & $79.3 \pm 7.8$ \\
Blood pressure & $125.5 / 82.3( \pm 1.7 / 8.4)$ & $120.8 / 79.8( \pm 7.2 / 8.1)$ \\
\hline \hline
\end{tabular}

\section{B. Experiment Procedure}

For all of the 30-odd volunteers, the experiment subjects were selected after MEQ (Morningness-Eveningness Questionnaire) examination and pre-test (blood pressure, pulse, obesity, and physiological signals). Four males and four females, whose life styles and sleep habits were judged to be similar on the basis of preliminary tests and physiological signals (Electroencephalography; EEG), were selected. The states of these subjects are shown in Table I . Preliminary training was given to subjects before commencing the experiment to make them fully understand the nature of the experiment. All the subjects wore summer uniforms $(0.33 \mathrm{Clo})$ to match standard clothing and covered themselves with summer bed blankets (made of rayon and synthetic fibers) on single beds.

The experimental subjects who participated in the research were made sleep after adapting their environment to the same conditions as those of the experiment for 2 days, considering the burden of their sleep environment and sensors.

\section{C.Data Collection \& Analysis}

An environmental chamber with a width $2.7 \mathrm{~m}$, a length of

$4.6 \mathrm{~m}$, and a height of $2.7 \mathrm{~m}$ was arranged for the sleep environment and the 4 walls, ceiling and floor were insulated

with $50 \mathrm{~mm}$ insulation materials. The set temperature and humidity were controlled by a thermo-hygrostat. To block noise transmitted from the thermo-hygrostat into the sleep area, a low noise fan was used to maintain a noise level less than $32 \mathrm{~dB}(\mathrm{~A})$.

In Fig. 2, a brief diagram of the testing equipment and the appearance of the subjects while sleeping with sensors attached are illustrated. Room temperature measurement for the test

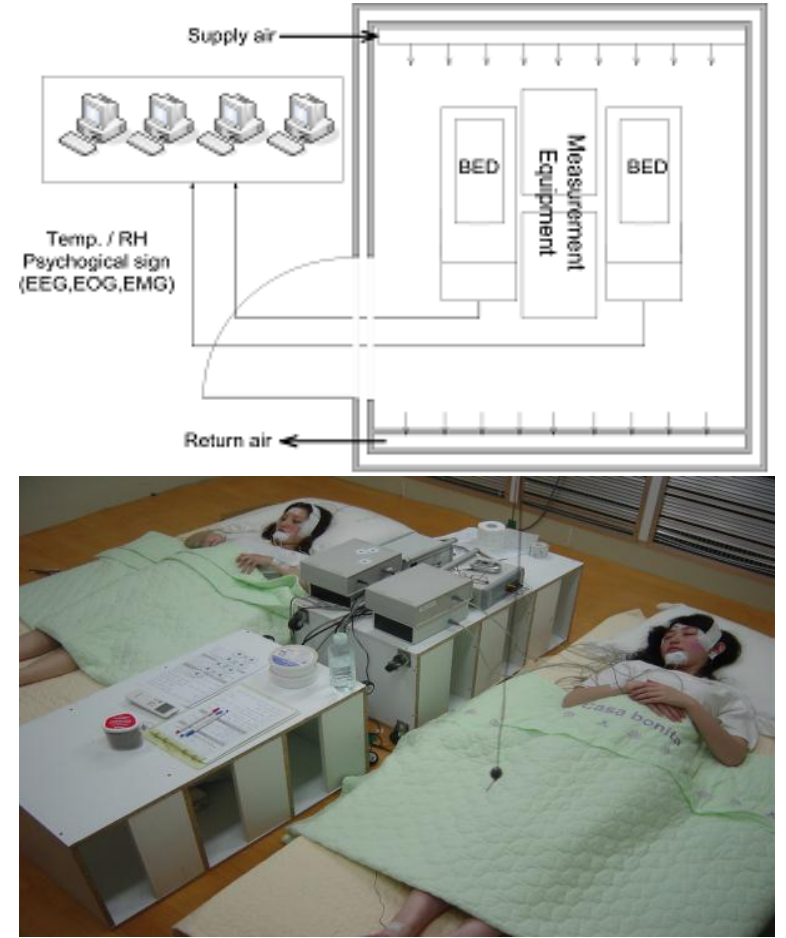

Fig. 2 Brief diagram of the testing equipment and the appearance of the subjects

environment was carried out using $\mathrm{T}$ shaped thermocouples at $30 \mathrm{~cm}$ above the mattress. Rectal temperature was measured in 1 subject among the 2 experimental subjects by inserting a dedicated sensor into the rectum using core body temperature measurement equipment (NKYSI NYLogger). Physiological signals such as EEG (Electroencephalography), ECG (Electrocardiogram), EOG (Electrooculography), EMG(Electromyography), etc. were measured during the sleep of the subjects, who were lying on beds with electrodes attached. Table II shows the measurement items and the testing equipment. For the assessment of sleep environment or sleep quality, Total Sleep Time(TST), the time spent in sleep stages 3, which is called SWS (slow wave sleep), and rate of sleep stages 3 against total sleep time (SWS/TST) were observed. TST was calculated according to the proportion of wakefulness during sleep, thus the higher the TST, the longer the wakefulness state and the less sleep time. Sleep efficiency was calculated as the rate of deep sleep among total sleep time and it indicates sufficient deep sleep time.

TABLE II

\begin{tabular}{c|c|c}
\hline \multicolumn{2}{c}{ MEASUREMENT ITEMS AND EQUIPMENT } \\
\hline \multirow{3}{*}{ Man's body } & Rectal temperature & Equipment \\
\cline { 2 - 3 } & EEG / EOG & LAXTHA NYLogger \\
\cline { 2 - 3 } & EMG/ECG & HANBYUL Meditech \\
\hline \multirow{2}{*}{$\begin{array}{c}\text { Indoor } \\
\text { environment }\end{array}$} & Temperature & YOKOGAWA MV200 \\
\cline { 2 - 3 } & Noise & Testo 815 \\
\hline
\end{tabular}




\section{RESULTS AND DISCUSSION}

Table III shows a comparison of sleep efficiency, the time spent in each sleep stage, and the total sleep time during temperature compensation section (1:00 2:00) of 60 min. under Case A and Case B conditions after sleep onset (00:00 1:00) during which the temperature change conditions were the same. Though the difference in total sleep time under Case A and Case B conditions was minimal, during sleep stages 3, a greater period of time was spent $(25.7 \pm 13.5$ minutes $)$ under Case A condition than was the case with Case B condition (19.4 \pm 9.6 minutes). Sleep efficiency was higher under Case A condition $(44.3 \pm 21.8 \%)$ than Case B condition $(33.4 \pm 15.6 \%)$ by around $11 \%$ during sleep stages 3 of total sleep time. Therefore, Case A condition in which the temperature is increased rapidly to $28^{\circ} \mathrm{C}$ after $60 \mathrm{~min}$. of sleep onset, during which the room temperature is lowered, was found to be more favorable for deep sleep than Case B condition, during which room temperature increases gradually for $60 \mathrm{~min}$.

TABLE II

AVERAGE AND DEVIATION OF SLEEP EFFICIENCY, TIME SPENT DURING EACH SLEEP STAGE, AND TOTAL SLEEP TIME ( $\mathrm{N}$ : NUMBER OF SUBJECT)

\begin{tabular}{|c|c|c|c|c|c|c|c|}
\hline \multirow{2}{*}{ Mod } & & \multirow{2}{*}{$\begin{array}{c}\text { Sleep } \\
\text { Efficiency } \\
{[\%]}\end{array}$} & \multicolumn{4}{|c|}{ Sleep stage [min.] } & \multirow{2}{*}{$\begin{array}{c}\text { Total } \\
\text { sleep time } \\
\text { [min.] }\end{array}$} \\
\hline & & & Wake & REM & Stage 1-2 & Stage 3 & \\
\hline \multirow{2}{*}{$\begin{array}{c}\text { Case A } \\
(\mathrm{N}=8)\end{array}$} & Mean & 44.3 & 2.8 & 2.6 & 28.9 & 25.7 & 57.3 \\
\hline & Std. & 21.8 & 3.5 & 2.2 & 11.1 & 13.5 & 3.5 \\
\hline \multirow{2}{*}{$\begin{array}{c}\text { Case B } \\
(\mathrm{N}=8)\end{array}$} & Mean & 33.4 & 2.2 & 2.8 & 35.6 & 19.4 & 57.8 \\
\hline & Std. & 15.6 & 4.8 & 2.9 & 9.7 & 9.6 & 4.8 \\
\hline
\end{tabular}

Fig. 3 and Fig. 4 show a comparison of sleep efficiency and the time spent in each sleep stage between male and female subjects for the temperature compensation section, during which the room temperature is raised. For time spent in each sleep stage, a longer time spent in deep sleep stages (stages 3) was observed in female subjects, whereas in case of male subjects, light sleep stages (stages 1-2) were longer.

Sleep efficiency was higher in both male and female subjects under Case A condition, during which temperature compensation was done more rapidly than under Case B condition, with a more prominent effect in female subjects as their sleep efficiency increased by $15 \%(59.0 \pm 21.7 \%$ vs. $44.2 \pm 11.3 \%$ ) when compared to the increase of sleep efficiency of $7 \%$ on the part of male subjects under Case A condition(Fig. 4). Therefore, rapid temperature increase during temperature compensation was found to result in higher sleep efficiency and it was more effective for female subjects than male subjects.

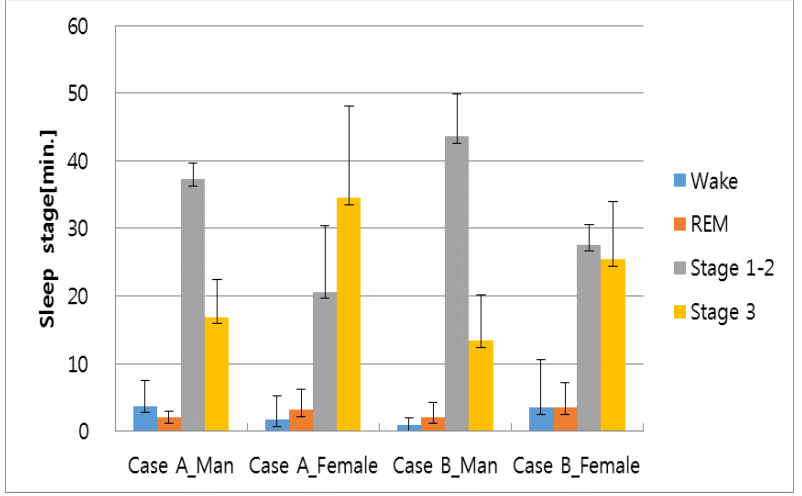

Fig. 3 Comparison of time spent per different sleep stage in male and female subjects during the temperature compensation period

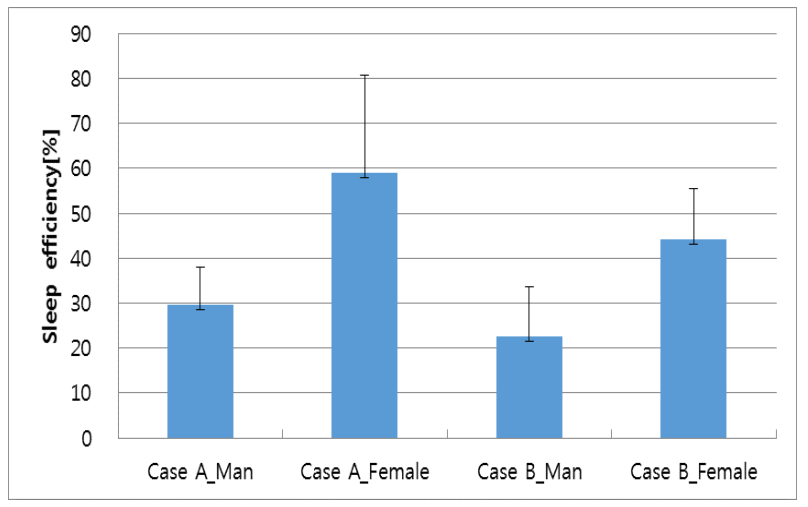

Fig. 4 Comparison of sleep efficiency in male and female subjects during the temperature compensation period

Fig. 5 shows rectal temperature changes as core body temperature in male and female subjects as per room temperature changes during sleep onset. In case of male subjects, rectal temperature started decreasing at elapse of 40 min. after sleep onset and was maintained after 110 minutes. Whereas the rectal temperature of female subjects started dropping at the time of sleep and it was maintained after 100 min. of sleep. The average rectal temperature drop (average value of first and last $5 \mathrm{~min}$.) during room temperature drop time was less in male $\left(0.1^{\circ} \mathrm{C}\right)$ than in female $\left(0.2 \sim 0.3^{\circ} \mathrm{C}\right)$ subjects, but it showed similar trends in both male as well as female subjects, with a value $0.2{ }^{\circ} \mathrm{C}$ during the temperature compensation section. Therefore a cool feeling due to a temperature drop in core body was judged to induce comfortable sleep during sleep onset. However, though rectal temperature showed dropping trends over a certain duration, even during the temperature compensation section, during which room temperature is increased, the body temperature of the subjects was above 36.0 ${ }^{\circ} \mathrm{C}$, which is the lower limit of normal body temperature due to temperature compensation. It is thus suggested that during sleep onset, the temperature compensation condition of increasing room temperature after a room temperature drop is an effective sleep control process, by preventing hypothermia while maintaining core body temperature, especially for females whose core body temperature is lower than that of males. 


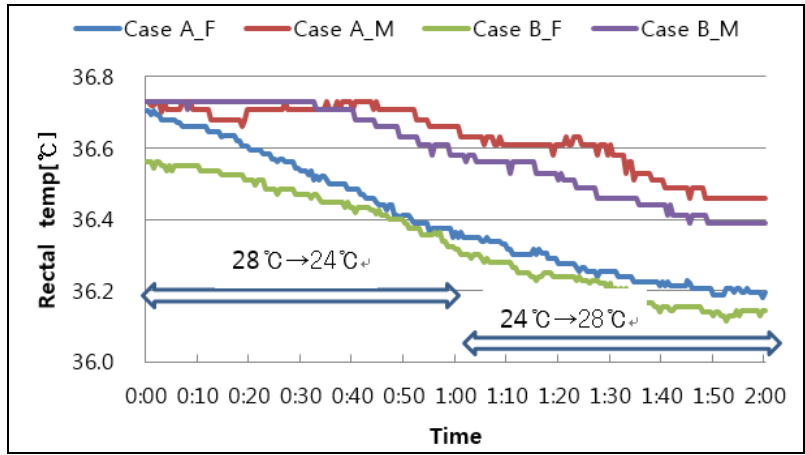

Fig. 5 Change in rectal temperature under Case A and Case B conditions

\section{CONCLUSION}

This experiment, which aimed to aid in the development of a temperature compensation control process against temperature drop during sleep onset to induce deep sleep, was performed and the conclusions that were drawn are as listed below:

1) During temperature compensation (for $60 \mathrm{~min}$.), sleep efficiency under the Case A control process, during which the temperature compensation occurred more rapidly, was higher than that under the Case B control process by around $11 \%$.

2) Sleep efficiency during temperature compensation was higher in female subjects and the same was higher by around 7 15\% in both male and female under the Case A condition, during which temperature compensation was carried out more rapidly than was the case under the Case B condition, during which temperature compensation was done slowly.

3) It is suggested that temperature a compensation condition could be an effective sleep control process during sleep onset, by preventing hypothermia while maintaining core body temperature, especially for females whose core body temperature is lower than that of males.

\section{REFERENCES}

[1] Kazue Okamoto-Mizuno, Kazuyo Tsuzuki, Koh Mizuno and Tatsuya Iwaki, "Effect of partial humid heat exposure during different segments of sleep on human sleep stage and body temperature", Physiology \& Behavior, 2005, Vol. 83, pp. 759-765

http://dx.doi.org/10.1016/j.physbeh.2004.09.009

[2] D.G. Kim, J.S. Kum, "Evaluation of Thermal Comfort during Sleeping in Summer(On results of questionnaire before and after sleep)", Korean Journal of Air-Conditioning and Refrigeration Engineering, 2005, Vol.17, No.5, pp.404-409.

[3] D.G. Kim, J.S. Kum, J.I. Park, "Evaluation of Thermal Comfort during Sleeping in Summer(About mean skin temperature and physiological signals)", Korean Journal of Air-Conditioning and Refrigeration Engineering, 2006, Vol.18, No.1, pp.1-6.

[4] N.B. Lee, J.J. Im, J.S. Kum, K.H. Lee, H.S. Choi, "Evaluation of Sleep Comfort for Indoor Thermal Environment based on the Physiolocical Signal Analysis", Korean Journal of The Science of Emotion \& Sensibility, 2000, Vol. 3, No.2, pp.75-84.

[5] D.G. Kim, J.S. Kum, S.H. Kim, "Evaluation of Thermal Comfort during Sleeping in Summer(About indoor air temperature rise)", Korean Journal of Air-Conditioning and Refrigeration Engineering, 2006, Vol.18, No.7, pp.535-540.

[6] D.G. Kim, J.S. Kum, J.I. Park, "Evaluation of Thermal Comfort during Sleeping in Summer(Study on indoor temperature conditions for comfort sleep)", Korean Journal of Air-Conditioning and Refrigeration Engineering, 2007, Vol.19, No.4, pp.307-312.

[7] E.H. Haskell, J.W. Palca, J.M. Walker, R.J. Berger, H.C. Heller, The effects of high and low ambient temperature on human sleep stages, Electroencephalography and Clinical Neurophysiology 51(1981) 494-501.

http://dx.doi.org/10.1016/0013-4694(81)90226-1 